\title{
Anaesthetic Challenges of a Patient Undergoing Left Cardiac Sympathetic Denervation in Therapy of Long QT Syndrome
}

\author{
Asoka Sameera Bandara Wasala ${ }^{I^{*}}$, Anura Bandara Abeysundara ${ }^{1}$, Kuda Banda Galketiya ${ }^{2}$, \\ Manura Madukelum Lekamwattage ${ }^{3}$, Yelaruwan Kumara Amarasinghe ${ }^{4}$, \\ Theja Thilakshani Amarasiri ${ }^{5}$ \\ ${ }^{1}$ Lecturer, Consultant Anaesthetist, ${ }^{2}$ Professor of Surgery, University of Peradeniya, Sri \\ Lanka, ${ }^{3}$ Consultant Cardiac Electrophysiologist, ${ }^{4}$ Registrar in Anaesthesiology ${ }^{5}$ Senior \\ Registrar in Anaesthesiology, Teaching Hospital Kandy, Sri Lanka
}

\begin{abstract}
A patient with prolonged QT syndrome (PQTS), whose symptoms were not controlled on maximal medical therapy, was scheduled for left thoracoscopic sympathectomy. The anesthetic management is discussed.
\end{abstract}

Key words: prolonged QT syndrome, left cardiac sympathetic denervation

\section{Introduction}

PQTS is a disorder of myocardial ion channel conduction that results in impaired ventricular repolarization. Beta blockers remain the mainstay of treatment for patients with PQTS syndrome. However, left cardiac sympathectomy is being increasingly advocated as an effective treatment for patients who are refractory to medical therapy. Patients with PQTS are at risk of developing polymorphic ventricular tachycardia or "Torsades de Pointes". With careful preoperative preparation and by avoidance of factors that precipitate cardiac dysrhythmias during the peri operative period, patients can be safely anaesthetised for thoracic sympathectomy

\section{Case Presentation}

A 26-year-old female was diagnosed as having PQTS following cardiac electro physiological studies at the National Hospital, Colombo. She presented with dyspnoea on mild exertion and multiple syncopal attacks over a 4-year period. Medical therapy was commenced and she was

*Correspondence: Asoka Sameera Bandara Wasala

E mail: wasalaasb@gmail.com

(iD) https://orcid.org/0000-0002-1309-032X

Received: $11 / 03 / 2020$

Accepted: $13 / 10 / 2020$

DOI: http:/doi.org/10.4038/slia.v29i1.8548 transferred to National Hospital Kandy for follow up. Despite maximum medical treatment with bisoprolol and nicorandil, her symptoms continued and she also suffered two witnessed cardiac arrests. She was referred to the Teaching Hospital, Peradeniya, for left thoracic cardiac sympathetic denervation.

Her medical history was unremarkable except for the PQTS. Serum $\mathrm{Na}^{+}, \mathrm{K}^{+}, \mathrm{Ca}^{2+}$ and $\mathrm{Mg}^{2+}$ were within normal limits. A multi-disciplinary meeting concluded that unilateral surgical denervation of the cardiac sympathetic chain was the best course of treatment. The risks and benefits of surgery were explained to the patient and informed consent was obtained. On the recommendations of the cardiac electrophysiologist, bisoprolol and nicorandil were continued till the day of surgery.

The patient was premedicated with midazolam. In the operating room, standard ASA monitors and external self-adhesive defibrillator pads were applied. An 18G IV cannula was placed and $2 \mathrm{~g}$ of $\mathrm{MgSO}_{4}$ administered. General anaesthesia was induced with midazolam, fentanyl, propofol and atracurium. Endotracheal intubation was done with a single lumen PVC tube. Anaesthesia was maintained with isoflurane in oxygen and air. Ventilation was targeted to maintain an end tidal $\mathrm{CO}_{2}$ of approximately $35 \mathrm{mmHg}$. Tidal volumes were adjusted to keep intra-thoracic pressures at lowest possible level to facilitate the surgery. Nasopharyngeal temperature was monitored and IV fluids were administered through a fluid warmer.

The patient was placed in the right lateral position 
and all pressure points were padded. Thoracoscopic ports were inserted and a pneumothorax with $\mathrm{CO}_{2}$ to a pressure of $8 \mathrm{mmHg}$ was achieved. After identifying the sympathetic chain, it was divided between the $3^{\text {rd }}$ and $4^{\text {th }}$ thoracic ganglia. Proximal dissection was followed by excision of the inferior pole of the stellate ganglion. The surgery was uneventful and the patient was stable throughout. After recovery of neuromuscular function, she was extubated in the operating room. Anti-cholinergic medications were not administered in order to prevent cardiac dysrhythmias. The patient was admitted to the ICU and had an uneventful post-operative course. She was discharged home on bisoprolol. She remained symptom free without recorded cardiac events in the implantable loop recorder at 6 months follow-up.

\section{Discussion}

PQTS is a cardiovascular disorder of ventricular repolarization that may precipitate malignant ventricular dysrhythmias. Most notably, pleomorphic ventricular tachycardia (PVT) or "Torsades de Pointes". In the inherited form of the illness, mutations in the cardiac ionic channels are thought to be the primary aetiological mechanism. ${ }^{1,4}$ The electrocardiogram demonstrates a prolonged QT interval of greater than $>440 \mathrm{~ms}$ in men or > $460 \mathrm{~ms}$ in women. Acquired PQTS can be due to side effects of medications such as antiarrhythmic agents, antibiotics, antihistamines, antidepressant, and prokinetics. ${ }^{1,2,4}$ Other causes of acquired PQTS include hypokalaemia, hypomagnesaemia and hypocalcaemia. ${ }^{1,2,4}$ PQTS can also be precipitated by a myocardial infarction, hypothyroidism or hypothermia. ${ }^{1,2}$ Patients are often commenced on beta blockers for control of dysrhythmias. Patients may also be implanted with cardioverter defibrillators (ICD) or permanent pacemakers. ${ }^{3}$ Although beta blockers remain the main stay of therapy, it is not entirely effective in preventing PVT and sudden cardiac death. In patients who continue to have dysrhythmias despite maximal medical therapy, cardiac sympathetic denervation has been shown to reduce their frequency and severity. 5,6

The principal challenges that the anaesthesiologist faces are preventing and managing PVT. Factors known to precipitate PVT include drugs, light anaesthesia, hypertension, bradycardia, tachycardia, hypoxaemia, hypocapnia, hypercapnia, hypothermia and excessive sympathetic tone ${ }^{2}$. It is important to select medications with minimum effect on the QT interval. Propofol can be used safely to induce anaesthesia due to its minimum effect on the QT interval. ${ }^{7,9}$ Short acting opioids such as fentanyl, alfentanyl and remifentanyl can be used to blunt the intubation response. ${ }^{7,8,10}$ Succinylcholine should be avoided as it increases serum potassium and has effects on the autonomic nervous system. Vecuronium, atracurium, cisatracurium, and rocuronium have minimal effect on ventricular reploarisation. ${ }^{7,8,10}$ When available, the use of Sugammadex to reverse neuromuscular block induced by rocuronium is particularly advantageous as atropine and neostigmine use can be avoided. Alternately atracurium can be used with neuromuscular monitoring, to enable recovery without reversal agents. ${ }^{8}$ Total intravenous anaesthesia (TIVA) with propofol and remifentanil, with depth of anaesthesia monitoring, would be ideal, as all halogenated inhalation agents increase the QT interval. ${ }^{8,9}$ However, isoflurane has been safely administered according to several case reports. ${ }^{8,10}$ Adequate analgesia is vital to reduce the sympathetic stimulation and morphine has been proven to be safe. ${ }^{10}$

Intra thoracic pressure should be kept at minimal level to facilitate surgery with a single lumen endotracheal tube. Hypercapnoea can be prevented by adjusting the minute ventilation. Metaraminol and phenylephrine have been used safely to treat hypotensive episodes during anaesthesia. However, due to the sympathomimetic action, adrenaline, noradrenaline, isoprenaline and ephedrine should only be used when absolutely necessary. ${ }^{8,10}$

In conclusion, Patients with both congenital and acquired PQTS are at risk of developing malignant ventricular dysrhythmias. Preoperative beta blockade minimizes the risk of PVT. Left cardiac sympathetic denervation has been shown to reduce incidence and severity of cardiac dysrhythmias. Good pre-operative preparation, the use of safe anaesthetic drugs and techniques, all contribute towards a satisfactory outcome from thoracoscopic sympathetic denervation.

\section{References}

1. Booker PD, Whyte SD, Ladusans E J. Long QT syndrome and anaesthesia. BJA, 2003; 90(3): 349366. 
https://doi.org/10.1093/bja/aeg061

PMID: 12594150

2. Hunter JD, Sharma P, Rathi S. Long QT syndrome. Continuing Education in Anaesthesia Critical Care \& Pain 2008; 8(2): 67-70. https://doi.org/10.1093/bjaceaccp/mkn003

3. Groh WJ, Silka M J, Oliver RP, Halperin BD, McAnulty JH, Kron. Use of implantable cardioverter-defibrillators in the congenital long QT syndrome. American Journal of Cardiology. 1996; 78: 703 - 706. https://doi.org/10.1016/s0002-9149(96)00403-3 PMID: 8831415

4. Sherif E, Boutjdir TG. Acquired long QT syndrome and torsade de pointes. Pacing and Clinical Electrophysiology 2018; 41 (4): 414-421. https://doi:10.1111/pace.13296 PMID: 29405316

5. Moss AJ, Zareba W, Hall WJ, Schwartz PJ, Crampton RS, Benhor in J, G. Vincent M, Locati EH, Priori SG, Napolitano C, Medina A, Zhang L, Robinson JL, Timothy K, Towb in JA, and Andrews ML. Effectiveness and limitations of $\beta$ blocker therapy in congenital long-QT syndrome. Circulation 2000; 101: 616 - 23 https://doi.org/10.1161/01.cir.101.6.616 PMID: 10673253

6. Schwartz PJ, Priori SG, Cerrone M, Spazzolini C, Odero A, Napolitano C, Bloise R, De Ferrari GM, Klersy C, Moss AJ, Zareba W, Robinson JL, Hall WJ, Brink PA, Toivonen L, AE Epstein, Li C.Left cardiac sympathetic denervation in the management of high-risk patients affected by the long-QT syndrome. Circulation 2004; 109: 182633 https://doi.org/10.1161/01.cir.0000125523.14403. $\underline{1 \mathrm{e}}$

PMID: 15051644
7. Kies S J, Pabelick CM, Hurley HA, White RD, Ackerman MJ. Anesthesia for Patients with Congenital Long QT Syndrome. Anesthesiology 2005;102:204-210

https://doi.org/10.1097/00000542-200501000$\underline{00029}$

PMID: 15618804

8. Lischke V, Wilke HJ, Probst S, Behne M, Kessler P. Prolongation of the QT-interval during induction of anesthesia in patients with coronary artery disease. Acta Anaesthesiologica Scandinavica $\quad 1994 ; \quad 38$ : $144-8$ https://doi.org/10.1111/j.13996576.1994.tb03856.x PMID: 8171949

9. Kleinsasser A, Loeckinger A, Lindner KH, Keller C, Boehler M, Puehringer F. Reversing sevoflurane-associated Q-Tc prolongation by changing to propofol. Anaesthesia 2001 ; 56: 24850

https://doi.org/10.1046/j.1365-

2044.2001.01717.x

PMID: 11251432

10. O'Hare M, Maldonado J. Munro J, Ackerman MJ, Ramakrishna H, Sarajja D. Ackerman Perioperative management of patients with congenital or acquired disorders of QT interval. BJA 2018; 120(4):

629-644 https://doi.org/10.1016/j.bja.2017.12.040 PMID: 29576105 